\title{
A Study of the Quantum Correlations in a Tavis-Cummings System
}

\section{Estudio de correlaciones cuánticas en un sistema Tavis-Cumming}

\author{
D. Madrid-Úsuga $\mathrm{a}^{*}$ \\ P. Villamilb
}

Fecha de Recepción: 03 - sep - 2017

Fecha de Aceptación: 06 - dic - 2017

\begin{abstract}
The quantum correlations of two qubits in a microcavity with dissipation and a single quantized mode of the electromagnetic field are studied. In order to study our physical system, the Tavis-Cummings model and the formalism of the master equation of the density operator under the Born-Markov approximations will be used. The cavity mode will be coupled with an external pulsed laser and the master equation of the density operator that describes the evolution of the system will be solved numerically. The concurrence, the quantum mutual information, and the quantum discord are determined for the system of two qubits. The correlations are studied as a function of the dimensionless quantity $\tau=g t$ when the intensity of the laser cavity coupling varies, and two initial conditions are considered in the weak coupling regime in which the dynamic evolution of these correlations was studied.
\end{abstract}

Keywords: Open Quantum Systems, Master Equation, Tavis-Cummings Model, Quantum Correlations, Concurrence, Mutual Information, Quantum Discord.

\section{Resumen}

Se estudian las correlaciones cuánticas de dos qubits en una microcavidad con disipación y un solo modo cuantificado del campo electromagnético interactuado con ella. Para estudiar nuestro sistema físico, se utiliza el modelo de Tavis-Cummings y el formalismo de la ecuación maestra del operador de densidad bajo las aproximaciones de Born-Markov. El modo de cavidad estará acoplado con un láser pulsado externo y la ecuación maestra del operador de densidad que describe la evolución del sistema será resuelta numéricamente. La concurrencia, la información mutua cuántica y la discordancia cuántica se determinan para el sistema de dos qubits. Las correlaciones se estudian como una función de la cantidadadimensional $\tau$ = gt cuando la intensidad de acoplamiento cavidad láser varía, y se consideran dos condiciones iniciales en el régimen de acoplamiento débil en los que se estudió la evolución dinámica de estas correlaciones.

Palabras clave: Sistemas Cuánticos Abiertos, Ecuación Maestra, Modelo Tavis-Cummings, Correlaciones Cuánticas, Concurrencia, Información Mutua, Discordia Cuántica.

\footnotetext{
a Departamento de Física, Universidad de Sucre, A.A. 406, Sincelejo, Colombia.

*Correo electrónico: duvalier82@gmail.com

bDepartamento de Física, Universidad de Sucre, A.A. 406, Sincelejo, Colombia.
} 


\section{INTRODUCCIÓN}

The quantum computing theoretically studies systems that make direct use of the phenomena of quan tum mechanics, such as superposition and entanglement, to perform operations on data [1]. Considering that the common digital computation requires that the data be coded in binary digits (bits), each of which is always in one of two defined states ( 0 or 1$)$, quantum computation uses qubit bits, which can Being in a superposition of states. The field of quantum computation began with the work of Paul Benioff [2] and Yuri Manin in 1980 [3], Richard Feynman in 1982 [4] and David Deutsch in 1985 who outlined the basic principles of quantum computation and proposed Idea of a quantum computer [5]. As of 2016, the development of real quantum computers is still in its infancy. Experiments have been carried out in which quantum computational operations were performed on a very small number of quantum bits [6]. Practical and theoretical investigations continue and many groups worldwide investigate quantum computation in an effort to develop quantum computers that can assist in the development of existing problems that have so far as in the area of cryptanalysis [7].

The potential of use in quantum computation motivates the detailed study of two-level atoms, in particular the atoms in the optical cavities where these can interact strongly with the individual photons. The system of a two-tiered atom coupled to a single cavity mode is known as the JaynesCummings model; Models of more complicated systems with multi-level atoms can be developed using their basic ideas, such as the Dicke model that corresponds to a set of two-level $\mathrm{N}$ atoms that may be interacting with each other [8].

In this work we will study the non-classical properties of the Tavis-Cummings system, which considers two qubits that interact with a single cavity mode and consider an external pulse that interacts with the unique cavity mode. Knowledge of the behavior of such systems may lead some day to applications in the field of quantum computing, or in related fields. The knowledge of how twolevel and multi-level atoms (more suitable for real systems) behave compared to idealized systems has direct relevance to know if such multilevel atoms, for example, can be used as qubits to encode quantum information.

Some of these properties is quantum entanglement $(\mathrm{QE})$ is a property of quantum systems that exhibit correlations that can not be explained classically and their discovery marks a big difference between the classical and quantum behavior of composite systems. however, QE is not the only useful quantum correlation for the quantum processing of information. Quantum discord (QD), initially introduced by Ollivier and Zurek [9] and later by Henderson and Vedral [10], is defined as the difference between the mutual information of the system and the information obtained after measuring one of the parts of the system. Quantum discord gives a measure of the quantum correlations and in particular of how much a system is disturbed when it is measured and the information obtained from it. QD measures quantum correlations of a more general type than entanglement. Curiously, it has been theoretically and experimentally demonstrated that separable states supply an increase in the computational speed in comparison with the classical states in some models of quantum computation [11].

Entanglement and quantum discord are of great interest because they constitute one of the basic tools for studying the effects in atoms located in cavities, in quantum optics, in trapped ions, in the optomechanics of cavities, etc. Correlations can be obtained, such as mutual information, concurrence, and non-classical correlations between the photons that leave a cavity and the cavity mode. These phenomena have been measured in atomic systems as well as in solid-state systems [12] Additionally, these correlations produce blocking of photons and tunneling, which can be used for the generation of single photons for the quantum processing of information [13] and high-precision detection and metrology, as well as the quantum simulation of many-body systems citecarusotto2009.

This article is organized as follows. In section II First we review the description of the model of Tavis- Cummings, in addition, it is considered an external laser coupled with a mode of the cavity. In Section III we present the definitions of concurrency and its relation to entanglement in quantum systems, Von Neumann's entropy and mutual 
information. We present the concept of quantum discord introduced by Ollivier and Zurek, which gives information of the quantum correlations present in a system. In Section IV, the dynamic evolution of interlacing, concurrency, mutual information and quantum discord for two different initial conditions in the weak coupling regime, and we conclude in section $\mathrm{V}$.

\section{THEORY}

\subsection{Hamiltonian of system}

The quantum system is made up of two qubits in the interior of a nanocavity with dissipation, which interact with a single quantized mode of the electromagnetic field with a frequency of $\omega_{c}$. The qubitcavity system is coherently impacted by a laser pulse with central frequency wl and intensity $\eta$. See Fig.1. In the dipolar approximations and those of the rotating wave, the Hamiltonian that describes the dynamics is given by $\widehat{H}_{S}$ :

$$
\widehat{H}_{S}=\widehat{H}_{0}+\widehat{H}_{A C}
$$

These Hamiltonians are expressed as:

$$
\begin{gathered}
H_{0}=\hbar \omega_{c} \widehat{a}^{\dagger} \widehat{a}+\hbar \widehat{\omega}_{1} \widehat{\sigma}_{+}^{1} \widehat{\sigma}_{-}^{1}+\hbar \widehat{\omega}_{2} \widehat{\sigma}_{+}^{2} \widehat{\sigma}_{-}^{2}, \\
H_{A C}=i \hbar g_{1}\left(\widehat{a}^{\dagger} \widehat{\sigma}_{-}^{1}-\widehat{a} \widehat{\sigma}_{+}^{1}\right)+i \hbar g_{2}\left(\widehat{a}^{\dagger} \widehat{\sigma}_{-}^{2}-\widehat{a} \widehat{\sigma}_{+}^{2}\right),
\end{gathered}
$$

In them, $\omega_{c}, \omega_{1}$, and $\omega_{2}$ are the frequencies of the cavity mode, of qubit 1 , and of qubit 2 , respectively. $\hat{a}$ and $\hat{a}^{\dagger}$ are the annihilation and creation operators associated with the single quantized mode of the electromagnetic field; $\widehat{\sigma}_{+}^{i}=\left|e^{i}\right\rangle\left\langle g^{i}\left|\mathrm{y} \widehat{\sigma}_{-}^{i}=\right| g^{i}\right\rangle\left\langle e^{i}\right|, \quad(i=1,2)$ are the Pauli operators of the ith qubit, and each possesses a transition frequency transition $\omega_{i}$. The coupling constant $g_{i}$ is the intensity of the cohe- rentinteraction between each qubit and the quantized mode of the electromagnetic field. If $\omega_{l} \sim \omega_{c}$, the laser-cavity interaction con be considered by means of a linear coupling constant $\varepsilon$ between the laser and the cavity mode. The Hamiltoniano of interaction between the quantized mode of cavity and the laser becomes

$$
\widehat{H}_{L C}=i \hbar \eta\left(\widehat{a} e^{-i \omega_{l} t}-\widehat{a}^{\dagger} e^{i \omega_{l} t}\right)
$$

where $\eta=\varepsilon \frac{c \varepsilon_{0}}{\omega_{l}} \sqrt{\frac{2 \pi c^{2}}{\hbar \omega_{c} V}}$ represents the intensity of the interaction. There $V=L^{3}$ is the volume of the cavity, $\varepsilon$ is the coupling constant between the laser and the cavity mode, and $c$ is the speed of light.

In the rotating wave approximation to the frequency $\omega_{l}$, the total Hamiltonian of the system is:

$$
\widehat{H}_{S}^{\prime}=\widehat{H}_{0}+\widehat{H}_{A C}+\widehat{H}_{L C}
$$

$$
\begin{aligned}
& \widehat{H}_{S}^{\prime}=\hbar \triangle_{c} \widehat{a}^{\dagger} \widehat{a}+\hbar \triangle_{1} \widehat{\sigma}_{+}^{1} \sigma_{-}^{1}+\hbar \triangle_{2} \widehat{\sigma}_{+}^{2} \sigma_{-}^{2} \\
& \quad i \hbar g_{1}\left(\widehat{a}^{\dagger} \widehat{\sigma}_{-}^{1}-\widehat{a} \widehat{\sigma}_{+}^{1}\right)+i \hbar g_{2}\left(\widehat{a}^{\dagger} \widehat{\sigma}_{-}^{2}-\widehat{a} \widehat{\sigma}_{+}^{2}\right) \\
& \quad i \hbar \eta\left(\widehat{a}+\widehat{a}^{\dagger}\right) .
\end{aligned}
$$

Here, $\Delta_{c}=\omega_{c_{-}}-\omega_{l}$ and $\Delta_{i}=\omega_{l}$ are the detuning of the cavity and of the ith qubit with respect to the frequency of the laser.

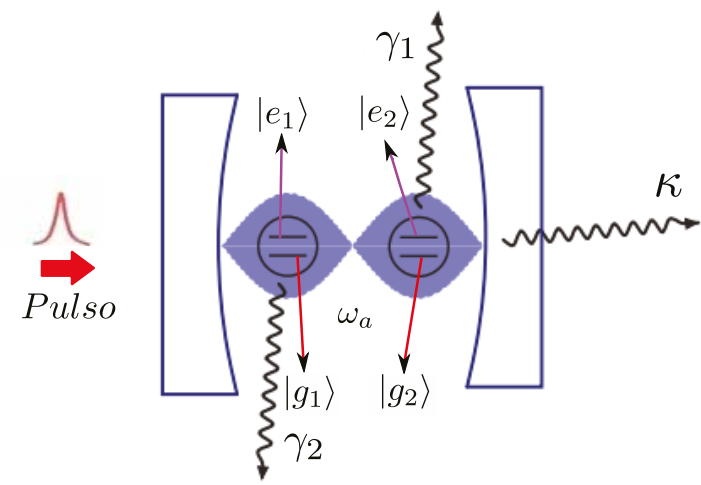

Figure 1. Representation of 2 qubits in the interior of a cavity with dissipation and a single quantized mode of the electromagnetic field with frequency $\omega$ a coupled with a pulsed laser of frequency $\omega 1, \kappa$ represents the rate of decay of the field of the cavity, and $\gamma_{i}$ is the rate of spontaneous emission of the ith qubit.

The dynamics of the qubit-cavity system are determined with the master equation of the density operator in the Lindblad form.

$\frac{d}{d t} \widehat{\rho}_{S}=\frac{1}{i \hbar}\left[\widehat{H}_{S}^{\prime}, \widehat{\rho}\right]-\frac{\kappa}{2} \widehat{\mathscr{L}}[\widehat{a}]-\frac{\gamma_{1}}{2} \widehat{\mathscr{L}}\left[\widehat{\sigma}^{1}\right]-\frac{\gamma_{2}}{2} \widehat{\mathscr{L}}\left[\widehat{\sigma}^{2}\right]$.

In this equation, $\widehat{\rho}$ is the density operator of the coupled qubit-cavity system. The Lindblad operator $\widehat{\mathscr{L}}[\widehat{x}]$ takes the form:

$$
\widehat{\mathscr{L}}[\widehat{x}]=\widehat{x}^{\dagger} \widehat{x} \widehat{\rho}+\widehat{\rho} \widehat{x}^{\dagger} \widehat{x}+\widehat{x} \widehat{\rho} \widehat{x}^{\dagger}
$$


and models the incoherent decay. Eq.(6) contains of 4 terms on the right of the equals sign. The first describes the coherent evolution of the qubit-cavity system, the second describes the rate of decay of the field of the cavity, and the third and fourth describe the processes of spontaneous emission of the ith qubit, $\kappa$ and $\gamma i$ are the rates at which photons escape across the mirrors of the cavity and the rate of decay due to spontaneous emission, respectively. The master equation is solved numerically using the program QuTip: Quantum Toolbox in Python, truncating the states of the photons at 10 . This method is completely quantum mechanical, and additional approximations besides those of Born-Markov are not carried out.

\section{QUANTUM ENTANGLEMENT AND QUANTUM DIS CORD}

The Hilbert space $\mathscr{H}$ of the compound system is given by the tensor product $\mathscr{H}_{Q_{1}} \otimes \mathscr{H}_{Q_{2}} \otimes \mathscr{H}_{C}$, where $\mathscr{H}_{Q_{1}}\left(\mathscr{H}_{Q_{2}}\right)$ are the Hilbert spaces of qubit 1 (qubit 2) and $\mathscr{H}_{C}$ is the Hilbert space of the cavity mode. The dynamics of the of 2-qubit system are obtained by taking the trace of the density operator $\widehat{\rho}_{S}$ with respect to the variables of the electromagnetic field of the cavity, $\widehat{\rho}_{12}=\operatorname{Tr}_{c}\left[\widehat{\rho}_{S}\right]$

The entanglement of a binary system can be quantified by means of the concurrence [14], which for an arbitrary mixed state is given by:

$$
C\left(\widehat{\rho}_{12}\right)=\max \left(\lambda_{1}-\lambda_{2}-\lambda_{3}-\lambda_{4}, 0\right)
$$

which $0 \leq C\left(\widehat{\rho}_{12}\right) \leq 1$ and $\lambda_{1} \geq \lambda_{2} \geq \lambda_{3} \geq \lambda_{4}$ being the eigenvalues of the matrix $\mathscr{R}$.

$$
\mathscr{R}=\widehat{\rho}_{12}\left(\widehat{\sigma}_{y}^{1} \otimes \sigma_{y}^{2}\right) \widehat{\rho}_{12}^{*}\left(\widehat{\sigma}_{y}^{1} \otimes \sigma_{y}^{2}\right)
$$

The concurrence $C=0$ indicates a separable, or non-entangled, state, and $C=1$ indicates a maximally entangled state. Quantum discord is the first measure of quantum correlations besides entanglement. The number of quantum and classical correla tions in a binary quantum system $\widehat{\rho}_{12}$ can be deter mined by means of the quantum mutual information and the quantum discord, as Oliver and Zurek [9] propose. For a two-qubit quantum system, the quantum discord is given as the difference between the quantum mutual information and the classical correlation, as follows [15]:

$$
\mathscr{Q}\left(\widehat{\rho}_{12}\right)=\mathscr{I}\left(\widehat{\rho}_{12}\right)-\mathscr{C}\left(\widehat{\rho}_{12}\right),
$$

$\mathscr{Q}\left(\rho_{12}\right)$ is the quantum discord, $\mathscr{I}\left(\rho_{12}\right)$ is the mutual information, and $\mathscr{C}\left(\rho_{12}\right)$ are the classical correlations. The mutual information of the two subsytems is expressed as:

$$
\mathscr{I}\left(\widehat{\rho_{12}}\right)=\mathscr{S}\left(\widehat{\rho}_{1}\right)+\mathscr{S}\left(\widehat{\rho}_{2}\right)-\mathscr{S}\left(\widehat{\rho}_{12}\right),
$$

where $S\left(\widehat{\rho}_{12}\right)=-\operatorname{Tr}\left(\widehat{\rho}_{12} \log \widehat{\rho}_{12}\right)$ is the von Neumann entropy of the quantum system and $\widehat{\rho}_{1}$ and $\widehat{\rho}_{2}$ the reduced density operators for the subsystems qubit 1 and qubit 2 , respectively. The classical correlations between the two subsystems qubit 1 and qubit 2 are given by $[11,15]$ :

$$
\mathscr{C}\left(\widehat{\rho}_{12}\right)=\mathscr{S}\left(\widehat{\rho}_{1}\right)-\min _{\left\{\Pi_{k}\right\}}\left[\mathscr{S}\left(\widehat{\rho}_{12} \mid\left\{\Pi_{k}\right\}\right)\right]
$$

The minimum is taken from the projective measurements $\left\{\Pi_{\mathrm{k}}\right\}$ as a whole, and $\mathscr{S}\left(\widehat{\rho}_{12} \mid\left\{\Pi_{k}\right\}\right)=\sum_{k} p_{k} \mathscr{S}\left(\widehat{\rho}_{k}\right)$ is the mutual conditional entropy of qubit 1 , given the complete measurements in qubit 2 , with $\widehat{\rho}_{k}=\operatorname{Tr}_{2}\left(\Pi_{k} \widehat{\rho}_{12} \Pi_{k}\right) / p_{k}$ and $p_{k}=\operatorname{Tr}_{12}\left(\widehat{\rho}_{12} \Pi_{k}\right)$.

With the initial conditions given for the system under study, this is transformed into states X, and one can calculate the quantum discord in the following way [16]:

$$
\begin{aligned}
\mathscr{Q}\left(\widehat{\rho}_{12}\right) & =\mathscr{S}\left(\widehat{\rho}_{2}\right)-\mathscr{S}\left(\widehat{\rho}_{12}\right) \\
& +\min _{\left\{\Pi_{k}\right\}}\left[\left.\mathscr{S}\left(\widehat{\rho}_{0}\right)\right|_{\theta_{1}},\left.\mathscr{S}\left(\widehat{\rho}_{12} \mid\left\{\Pi_{k}\right\}\right)\right|_{\theta_{2}, \theta_{3}}\right]
\end{aligned}
$$

So using Eq. (10) and (13), the degree of the quantum entanglement and the quantum discord is numerically calculated for our study system.

\section{NUMERICAL RESULTS AND ANALYSIS}

Initially, the evolution of the qubit-cavity system is considered without interaction with the pulsed laser. In order to simplify the problem, it is assumed that $\mathrm{g}_{1}=\mathrm{g}_{2}=\mathrm{g}$ and $\omega_{1}=\omega_{2}=\omega$, so the Hamiltonian of the qubit-cavity system will be:

$$
\begin{gathered}
\widehat{H}_{S}=\hbar \omega \widehat{a}^{\dagger} \widehat{a}+\hbar \omega \widehat{\sigma}_{+}^{1} \widehat{\sigma}_{-}^{1}+\hbar \omega \widehat{\sigma}_{+}^{2} \widehat{\sigma}_{-}^{2}+ \\
i \hbar g\left(\widehat{a}^{\dagger} \widehat{\sigma}_{-}^{1}-\widehat{a} \widehat{\sigma}_{+}^{1}\right)+i \hbar g\left(\widehat{a}^{\dagger} \widehat{\sigma}_{-}^{2}-\widehat{a} \widehat{\sigma}_{+}^{2}\right),
\end{gathered}
$$


and the master equation for the density operator of the system is:

$\frac{d}{d t} \widehat{\rho}_{S}=\frac{1}{i \hbar}\left[\widehat{H}_{S}, \widehat{\rho}\right]-\frac{\kappa}{2} \mathscr{L}[\widehat{a}]-\frac{\gamma_{1}}{2} \mathscr{L}\left[\sigma_{-}^{1}\right]-\frac{\gamma_{2}}{2} \mathscr{L}\left[\sigma_{-}^{2}\right]$,

Two initial states for the system under study have been considered like those presented in [11].

- $|\psi\rangle_{12 c}=\alpha|g e 0\rangle+\beta|e g 0\rangle+\gamma|g g 1\rangle$, here $|\alpha|^{2}+$ $|\beta|^{2}+|\gamma|^{2}$, for simplicity, $\alpha=\sin \theta \cos \varphi, \beta=$ $\sin \theta \cos \varphi, \gamma=\cos \theta(\theta \in[0, \overline{2}]$ y $\varphi \in[0, \pi])$ is chosen.

- $\rho_{12 c}=\left(a\left|\Psi^{+}\right\rangle\left\langle\Psi^{+}|+(1-a)| e e\right\rangle\langle e e|\right) \otimes|n\rangle\langle n|$, where $\left|\Psi^{+}\right\rangle=\frac{1}{\sqrt{2}}\{|g e\rangle+|e g\rangle\}$, corresponds to one of the maximally entangled Bell states.

In this article we will study the quantum correlations of an open quantum system. The system operator density equation (Eq. 16) takes into account different processes: coherent emission $\kappa$, which are the loss of photons through the cavity and spontaneous emission $\gamma$ of the qubits.By changing the values of the free parameters in the model of

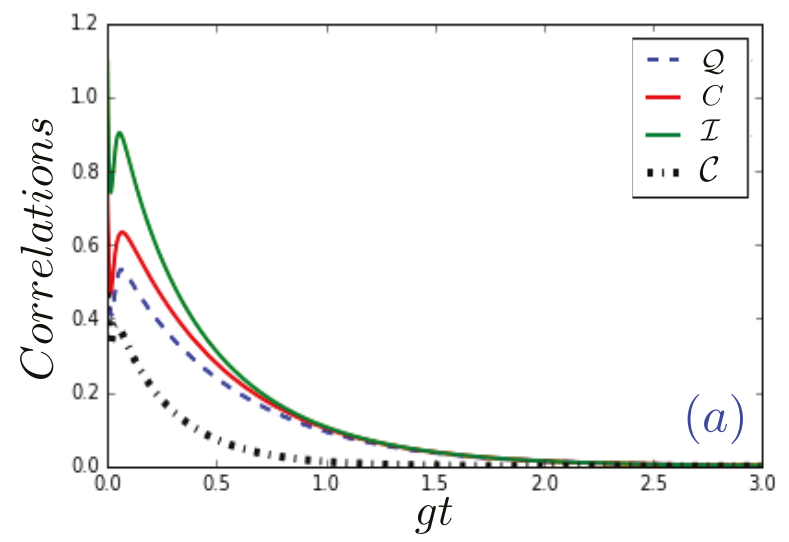

our study system, $\kappa$ and $\gamma$, two different regimes of the dynamics can be obtained: weak coupling and strong coupling between the quantized mode of the cavity and The qubits. In the first regime and that will be studied here the photons emitted by the qubits have an almost null chance of being reabsorbed by the medium. Thus the excitation of the qubits is dissipated. This regime is usually observed when the transition of qubit excitation occurs within the continuum of the photon state, resulting in a finite radiative lifetime of these excitations. In this case, the emission is governed by the golden rule of fermi, resulting in an exponential decay of the population of excited states [17].In the two figures, the concurrence reaches higher values than the quantum discord and decays more slowly. The single-mode electromagnetic field in the cavity has the responsibility for increasing the entanglement between the qubits, and as the photons in the cavity decrease so does the entanglement, until it completely disappears. Over the whole range of figures, the non-classical correlations are more robust than the classical correlations, and all of them coexist.

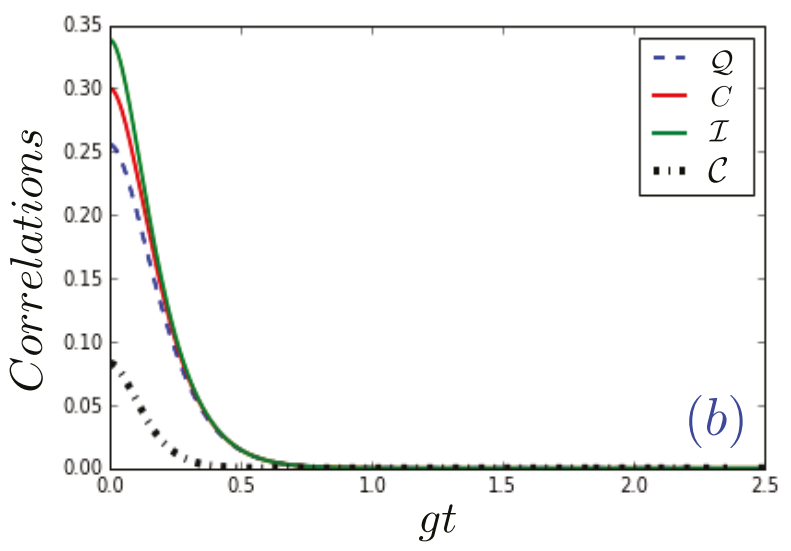

Figure 2. Quantum correlations for a qubit-cavity system as a function of gt, in the weak coupling regime. Quantum discord (dotted blue lines), concurrence (red lines), mutual Information (green lines), and classical correlations (dotted black lines) are shown. Graph (a) corresponds to the initial state $\mid \psi) 12 c, \theta=\pi / 3, \phi=\pi / 4$, Graph (b) corresponds to initial state $\rho 12 c$, with $a=0,3, g=4 \mathrm{GHz}, \gamma=0,25 \mathrm{GHz}$, and $\kappa=20 \mathrm{GHz}$. 
In Fig. 2 we present the dynamics of the correlations: quantum discord, mutual information, concurrence and classical correlations as a function of the $g t$ parameter, taking into account the decay effects Of the cavity and the spontaneous emission of the qubits. Fig. 2 occurs in the weak coupling regime where the parameters associated to the system satisfy the condition $16 g^{2}<(\kappa-$ $\gamma)^{2}$. In this regime the value of the parameter $(a=$ 0,3 ) is fixed and it is observed that a pronounced decay of the concurrence occurs until reaching a value of zero, because the effects caused by the doubly excited state For the case $|\psi\rangle_{12 c}$ favor that the system loses its correlations more quickly, in addition we observe that for initial condition $\rho_{12 c}$ shown in Fig. 2 (b) the system loses correlations in a shorter time interval than in the case of the first, Demonstrating that the correlations of the system depend on the state in which the system is initially prepared, this fact is also associated with the time in which the photons remain in the cavity, since their interaction with the qubits will be lower in this regime, as compared to a strong coupling regime.

To observe the effects caused by the increase in the intensity of the laser-cavity coupling on the quantum correlations, we consider four different values of this coupling. The dynamic evolution of the correlations for this case are shown in Fig. 3 and 4 associated with the initial conditions $|\psi\rangle_{12 c}$ and $\rho_{12 r}$ respectively. The laser-mode quantized interaction of the cavity with the qubits produces excitations and transitions between energy levels causing the temporal evolution of the system to show scenarios of great interest for different purposes. If the intensity of the laser coupling-cavity mode is increased, more photons are provided by the laser to the cavity and consequently increases the mean number of photons in the driven mode.
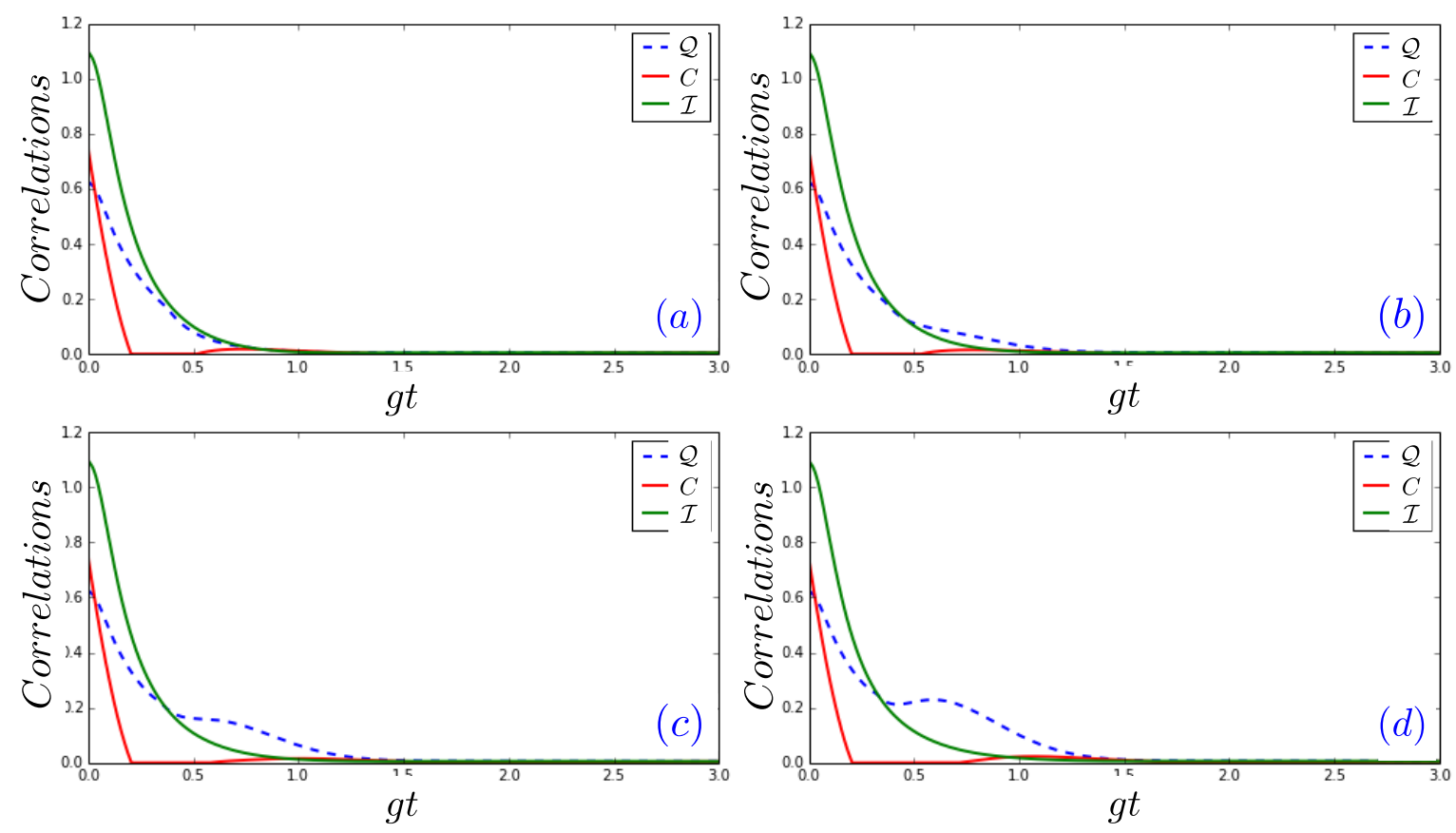

Figure 3. Dynamics of the quantum correlations as a function of gt, for the initial state $\psi 12 \mathrm{c}$, with $\theta=\pi / 3, \phi=\pi / 4, \mathrm{~g}=4 \mathrm{GHz}$, $\gamma=0,25 \mathrm{GHz}, \kappa=20 \mathrm{GHz}$, (a) $\Omega=1 \mathrm{GHz}$, (b) $\Omega=2 \mathrm{GHz}$, (c) $\Omega=3 \mathrm{GHz}$, and (d) $\Omega=4 \mathrm{GHz}$. In the graph, the quantum discord (dotted blue lines), the concurrence (solid red lines), and the mutual information (solid green lines) can be seen.)

Due to the small amount of time that the photons remain in the cavity, the opportunity for their interacting with the qubits is quite small, and oscillations in the correlations of Fig. 3 and 4 do not appear for the different intensities of coupling pulse-caviti. It should be kept in mind that the quantum discord plays an important part during the dissipative evolution of the qubits; it is not a matter of only the existence of quantum correlations, but also of the way in which they interrelate [18]. Exciting the system with a laser beam can be used as an additional means of dynamically controlling the given relation between the entanglement, the concurrence, and the quantum correlations present between the quantum dots. This fact could be used as an important re- 
source for the construction of non-conventional quantum protocols, as is studied in the paper [19].

Fig. 3 (a) shows that for the greatest part of the time of study of the system the entanglement almost disappears and the quantum discord rapidly approaches the value given by the total correlations or mutual information. It can be seen that this behavior changes as the intensity of the laser increases, as is shown in Fig. 3 (b), (c), and (d).
In Fig. 4, this behavior changes significantly, because the concurrence undergoes a sudden death and then revives for a longer period of time. The increase in the intensity of the laser favors the interaction of the photons with the qubits, and that is why the oscillations of the correlations tend to emerge in the system. This shows that the initial conditions of the system play an important part, because they can either favor or not favor interactions between the photons and the qubits in the cavity.
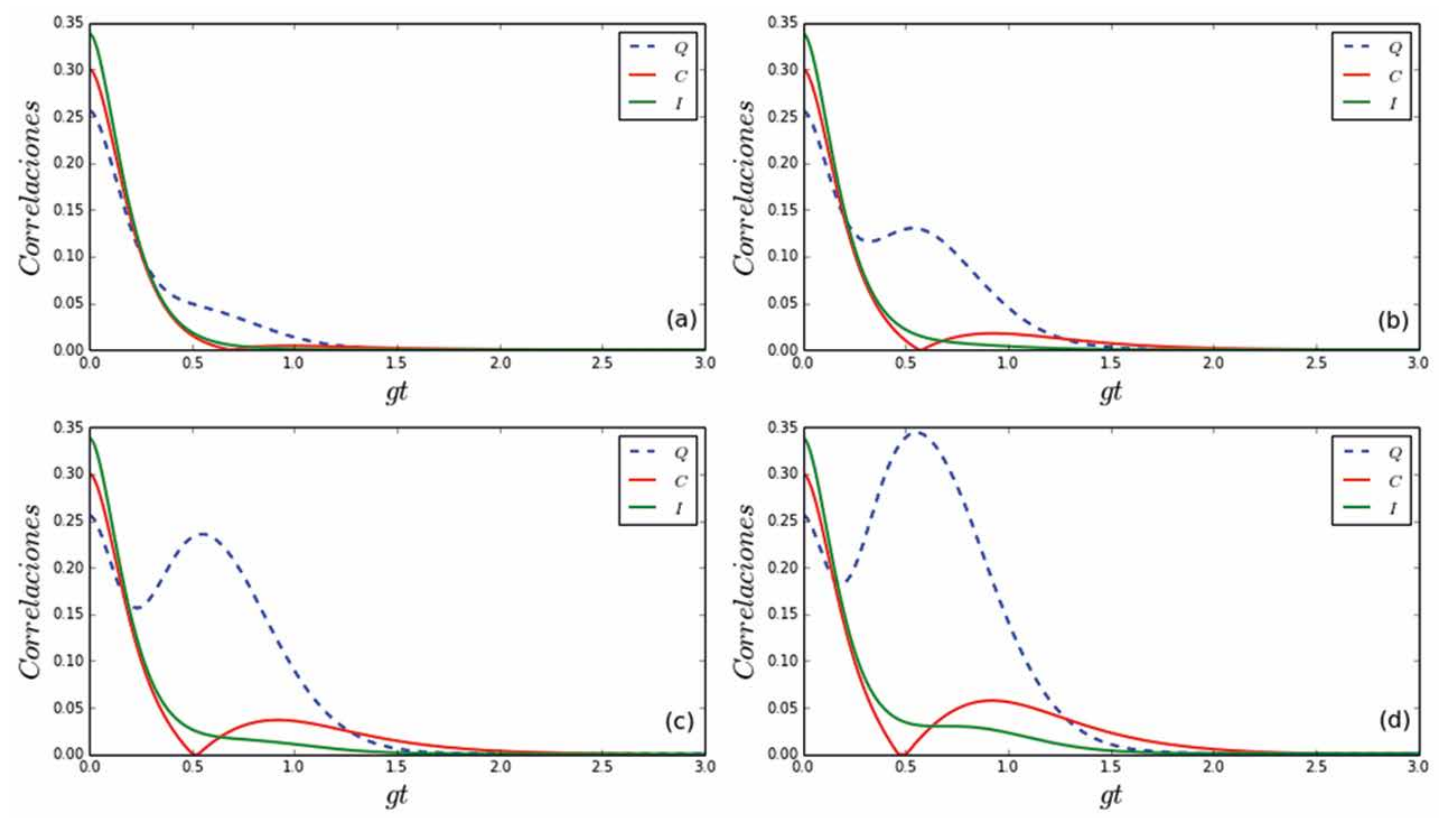

Figure 4. Dynamics of the quantum correlations as a function of gt, for the initial states $\rho 12 \mathrm{c}$, with $\mathrm{a}=0,3, \mathrm{~g}=4 \mathrm{GHz}, \gamma=0,25$ $\mathrm{GHz}, \kappa=20 \mathrm{GHz}$. (a) $\Omega=1 \mathrm{GHz}$, (b) $\Omega=2 \mathrm{GHz}$, (c) $\Omega=3 \mathrm{GHz}$, and (d) $\Omega=4 \mathrm{GHz}$. In the graph, the quantum discord (dotted blue lines), the concurrence (solid red lines), and the mutual information (solid green lines) can be seen).

Fig. 5 and 6 are presented with the aim of showing the changes in the correlations with the increase in the intensity of the laser pulse. Fig. 5 (a) and 5 (b) show that the quantum discord is the same for dif- ferent intensities of the laser within the range range $0,2 \geq g t \geq 1,7$. For the rest of the values of gt, the quantum discord increases with the increase in the intensity of the laser. These increases are more pro- nounced in the initial state $\rho_{12 c}$.

In Fig. 5 (b), which corresponds to initial state $\rho_{12 c}$, greater increases in the discord can be seen than in fig. 5 (a).

In Fig. 6 (a) and 6 (b), curves of the concurrence for four intensities of the laser pulse can be seen. Initially, the concurrence is the same for all of the intensities, and then it increases in value with the increase in the intensity of the pulse, but then all of them decrease to zero in the same gt. It can be seen that while the quantum discord increases, the concurrence decreases with the increase in the intensity of the inciding laser beam. 

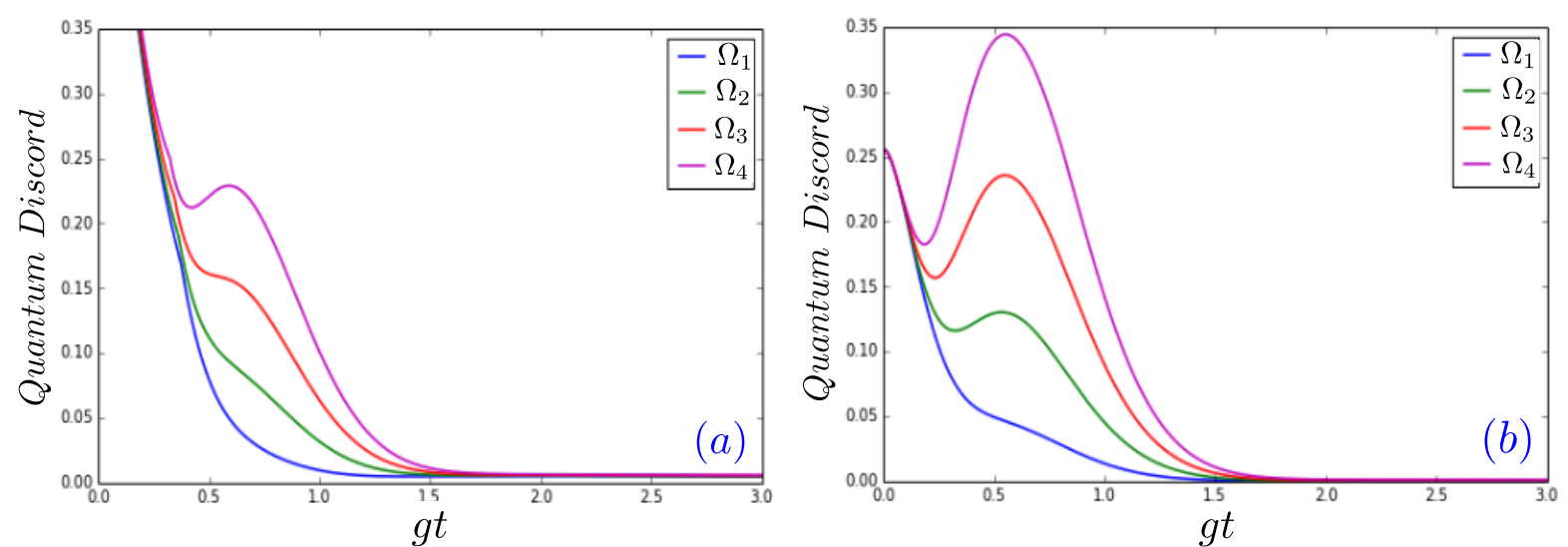

Figure 5. Quantum discord for a qubit-cavity system with incidence of a laser pulse, as a function of gt in the weak coupling regime. In the figures, the intensities $\omega_{1}=1 \mathrm{GHz}$ (blue lines), $\Omega_{2}=2 \mathrm{GHz}$ (green lines), $\omega_{3}=3 \mathrm{GHz}$ (red lines), and $\Omega_{4}=$ $4 \mathrm{GHz}$ (pink lines) are shown. (a) Corresponds to the initial state $1 \psi>12 \mathrm{c}$, with $\theta=\pi / 3$ and $\phi=\pi / 4$, and (b) Corresponds to initial state $\rho 12 \mathrm{c}$, with $\mathrm{a}=0,3, \mathrm{~g}=4 \mathrm{GHz}, \gamma=0,25 \mathrm{GHz}$, and $\gamma=20 \mathrm{GHz}$.
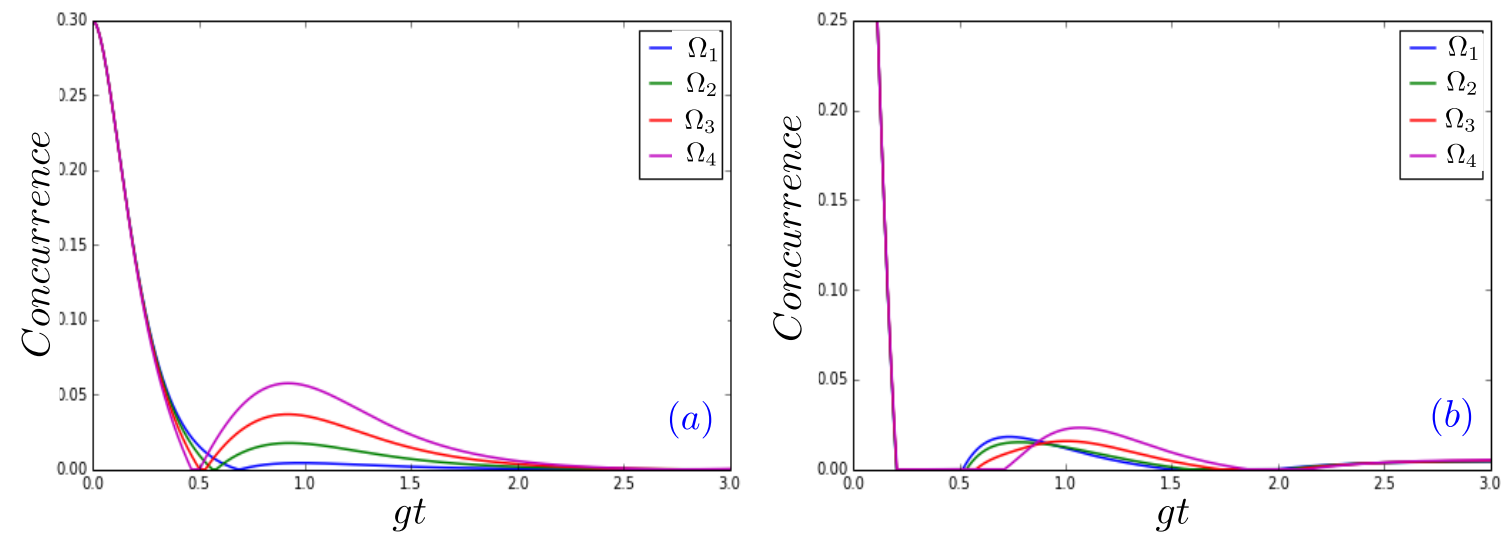

Figure 6. Concurrence for a qubit-cavity system with incidence of a laser pulse as a function of the gt in the weak coupling regime. Graph (a) corresponds to initial state $\mid \psi>12$ c, with $\theta=\pi / 3$ and $\phi=\pi / 4$, and Graph (b) corresponds to initial state $\rho 12 \mathrm{c}$, with $\mathrm{a}=0,3, \mathrm{~g}=4 \mathrm{GHz}, \gamma=0,25 \mathrm{GHz}$, and $\kappa=20 \mathrm{GHz}$. In the figures, the intensities $\Omega_{1}=1 \mathrm{GHz}$ (blue lines), $\Omega_{2}=2 \mathrm{GHz}$ (green lines), $\Omega_{3}=3 \mathrm{GHz}$ (red lines), and $\Omega_{4}=4 \mathrm{GHz}$ (pink lines) are shown.

\section{CONCLUSION}

In the development of the previous work we find that for the weak coupling regimes, considering two initial conditions given by: $\mid \psi>12 C=$ $\alpha|e g 0>+\beta| g e 0>+\xi \mid g g 1>$ and $\widehat{\rho}_{12 C}=a \mid \Psi^{+}>$ $<\Psi^{+}|+(1-a)| e e><e e|\otimes| 0><0 \mid$, Studied here, it has been found that the mean number of photons in the cavity shows an incoherent dynamics that is related to the time that the photons remain in the cavity. We observed that the oscillations present in the system better their behavior in the case of the initial condition $\widehat{\rho}_{12 C}$.
For the case in which the presence of a laser coupled to the mode of the studied radiation field is considered, we observe that the behavior of the studied correlations changes considerably as we can see in Fig. 5, And Fig. 5. In this regime the correlations show a behavior of a smooth curve that increases the amplitude of the correlation for the case of discord and mutual information but shows an opposite behavior for the case of the concurrence, for this last correlation we see that as we Is increased the laser- cavity coupling the degree of coupling of the system increases, the oscillations present in this regime are quantum oscillations of Rabbi that decay to a rat determined by the quality factor $\mathrm{Q}$ of the cavity. 
The response of the different correlations to the change in the intensity of the laser-cavity coupling is due to the change in the magnitude of the same. Quantum discordance presents the greatest response in comparison with the rest of the other correlations. It was found that the mutual information is greater than the concurrence in almost the entire work domain.

Quantum discord and quantum mutual information have been considered as quantifiers of correlations. This shows that care must be taken when analyzing the use of specific quantifiers to describe correlations in the system. Since the non-existence of interlacing in the system does not guarantee the non-correlation of the system given that there are correlations that can not be described by entanglement but are made by quantum discord as we can see in the case of Fig. $5 \mathrm{y}$ Fig. 6 where we observe that there is no entanglement in the system in the interval of about 0,5 and for times greater than 2 for condition 1 Fig. 6 (a) and the condition 2 Fig. 6 (b) In the interval of $(0,2-0,6)$ and times greater than 1.8 , however if we observe the quantum discord in figure 5, we see that the quantum discord is different from zero in the interval of $(0-1,5)$ lo Which shows that in this interval there are correlations in the system that can not be observed only by studying the interlacing throughout the interval.

\section{REFERENCIAS}

[1] N. Gershenfeld and I. L. Chuang, "Quantum computing with molecules," Scientific American, vol. 278, no. 6, pp. 66-71, 1998.

[2] P. Benioff, "The computer as a physical system: A microscopic quantum mechanical hamiltonian model of computers as represented by turing machines," Journal of statistical physics, vol. 22, no. 5, pp. 563591,1980 .

[3] Y. Manin, "Computable and uncomputable,"

[4] R. P. Feynman, "Simulating physics with computers," International journal of theoretical physics, vol. 21 , no. 6 , pp. 467488, 1982.
[5] D. Deutsch, "Quantum theory, the churchturing principle and the universal quantum computer," in Proceedings of the Royal Society of London A: Mathematical, Physical and Engi- neering Sciences, vol. 400, pp. 97-117, The Royal Society, 1985.

[6] D. Finkelstein, "Space-time structure in high energy interactions," 1969.

[7] E. Gershon, "New qubit control bodes well for future of quantum computing," Phys. org, 2014

[8] F. Dimer, B. Estienne, A. Parkins, and H. Carmichael, "Proposed realization of the dickemodel quantum phase transition in an optical cavity qed system," Physical Review $A$, vol. 75, no. 1, p. 013804, 2007.

[9] H. Ollivier and W. H. Zurek, "Quantum discord: a measure of the quantumness of correlations," Physical review letters, vol. 88, no. 1, p. 017901, 2001.

[10] L. Henderson and V. Vedral, "Classical, quantum and total correlations," Journal of physics A: mathematical and general, vol. 34, no. 35, p. 6899, 2001.

[11] Y.-J. Zhang, X.-B. Zou, Y.-J. Xia, and G.C. Guo, "Quantum discord dynamics in the presence of initial system-cavity correlations," Journal of Physics B: Atomic, Molecular and Optical Physics, vol. 44, no. 3, p. 035503, 2011.

[12] A. Faraon, I. Fushman, D. Englund, N. Stoltz, P. Petroff, and J. Vuckovic, "Coherent generation of nonclassical light on a chip via photoninduced tunneling and blockade," arXiv preprint arXiv:0804.2740, 2008.

[13] A. Faraon, A. Majumdar, and J. Vuckovic, "Generation of nonclassical states of light via photon blockade in optical nanocavities," Physical Review A, vol. 81, no. 3, p. 033838 , 2010. 
[14] W. K. Wootters, "Entanglement of formation of an arbitrary state of two qubits," Physical Review Letters, vol. 80, no. 10, p. 2245, 1998.

[15] S. Luo, "Quantum discord for two-qubit systems," Physical Review A, vol. 77, no. 4, p. 042303, 2008.

[16] X.-Q. Yan and Z.-L. Yue, "Correlated relation between quantum discord and entanglement of two-atom in thermal reservoirs," Journal of Atomic and Molecular Physics, vol.2014, 2014.

[17] J. Kasprzak, Condensation of exciton polaritons. $\mathrm{PhD}$ thesis, Université JosephFourier- Grenoble I, 2006.

[18] F. Fanchini, L. Castelano, M. Cornelio, and M. De Oliveira, "Locally inaccessible informa tion as a fundamental ingredient to quantum information," New Journal of Physics, vol. 14, no. 1, p. 013027, 2012.

[19] C. Zu, Y. Wang, X. Chang, Z. Wei, S. Zhang, and L. Duan, "Experimental demonstration of quantum gain in a zero-sum game," New Journal of Physics, vol. 14, no. 3, p. 033002 , 2012. 